\title{
A GENERALIZED LAPLACE-STIELTJES TRANSFORMATION
}

\section{GARNER MCCROSSEN}

1. Introduction. Gustav Doetsch ${ }^{1}$ has recently introduced a generalized Laplace transform of order $k, k \geqq 0$. It is the object of this paper to introduce a generalized Laplace-Stieltjes transform of order $k$.

We assume in what follows that $k$ is positive and integral. We also assume that $F$ denotes a complex-valued function of a real variable, which is defined, except perhaps on a set of isolated points, and is of bounded variation on every closed, finite interval $[0, T], T \geqq 0$. By $\{F\}$ we mean the class of all functions $F$ defined above. All integrals in this paper are to be interpreted as Riemann or Riemann-Stieltjes integrals. The symbol $\left({ }^{*}\right)$ will denote a convolution.

2. Definition of the transform. Let $s$ be complex. Let $M_{k}(s, t)$ be the Cesàro mean of order $k$ of

$$
\int_{0}^{t} e^{-s u} d F(u)
$$

i.e.,

$$
M_{k}(s, t)=\frac{k}{t^{k}}\left\{\left[\int_{0}^{t} e^{-s u} d F(u)\right] * t^{k-1}\right\} .
$$

If for some $s$ and some $k$

$$
\operatorname{Lim}_{t \rightarrow+\infty} M_{k}(s, t)
$$

exists, then this limit defines a value $f_{k}(s)$ of a function $f_{k}$ :

$$
f_{k}(s)=\operatorname{Lim}_{t \rightarrow+\infty} M_{k}(s, t) .
$$

We call $f_{k}$ the generalized Laplace-Stieltjes transform of order $k$ of $F$.

3. Existence and integral representations of the transform. We assume in what follows that $s_{0}$ is a fixed complex number. From the definition of $f_{k}\left(s_{0}\right)$ and the consistency of Cesàro summability we can assert the following:

Received by the editors March 15, 1955 and, in revised form, July 7, 1956.

${ }^{1}$ Gustav Doetsch, Handbuch der Laplace-Transformation, Basel, Verlag Birkhauser, pp. 311-352. 
THEOREM 1. If $f_{k}\left(s_{0}\right)$ exists and $N$ is a nonnegative integer, then, as $t \rightarrow+\infty$,

$$
\left[\int_{0}^{t} e^{-s_{0} u} d F(u)-f_{k}\left(s_{0}\right)\right] * t^{k+N-1}=o\left(t^{k+N}\right) .
$$

We now define

$$
H_{i}(t)=k\left[\int_{0}^{t} e^{-s_{0} u} d F(u)-f_{k}\left(s_{0}\right)\right] * t^{k-2+i}, \quad i=1,2 .
$$

Then, by Theorem 1 , if $f_{k}\left(s_{0}\right)$ exists,

$$
H_{i}(t)=o\left(t^{k-1+i}\right),
$$$$
i=1,2 \text {, }
$$

as $t \rightarrow+\infty$. We also define

$$
G(t)=\int_{0}^{t} e^{-s_{0} u} d F(u),
$$

so that we may now write (1) as

$$
H_{i}(t)=k\left[G(t)-f_{k}\left(s_{0}\right)\right] * t^{k-2+i}, \quad i=1,2 .
$$

In what follows we define an angular region $Q_{w}\left(s_{0}\right)$ to be the totality of all $s$ such that $\left|\arg \left(s-s_{0}\right)\right| \leqq w$, where $w$ is a fixed number satisfying $0 \leqq w<\pi / 2$.

Using the facts that $\left|s-s_{0}\right| \leqq R\left(s-s_{0}\right) / \cos w$ for every $s$ in $Q_{w}\left(s_{0}\right)$ $-\left\{s_{0}\right\}$ and that for every $\epsilon_{i}>0$ there exists, by (2), a $T_{i}$ such that $\left|H_{i}(t)\right|\left\langle\epsilon_{i} t^{k-1+i}\left(\cos ^{h-1+i} w\right) /(h-1+i)\right.$ ! whenever $t>T_{i}$, we can establish the following theorem:

THEOREM 2. Let $f_{k}\left(s_{0}\right)$ exist. Let $Q_{w}\left(s_{0}\right)$ be any fixed angular region. Then for any $\epsilon_{i}>0$ and nonnegative integer $h$ a $T_{i}$ exists such that uniformly in $Q_{w}\left(s_{0}\right)$

$$
\left|\left(s-s_{0}\right)^{h-1+i} e^{-\left(\diamond-s_{0}\right) t} H_{i}(t)\right|<\epsilon_{i} t^{k-h}
$$

whenever $t>T_{i}, i=1,2$.

THEOREM 3. If $f_{k}\left(s_{0}\right)$ exists and $R(s)>R\left(s_{0}\right)$, then $f_{k}(s)$ exists. Furthermore, $M_{k}(s, t)$ converges uniformly to $f_{k}(s)$ in every region $Q_{w}\left(s_{0}\right)$. If $R(s)>R\left(s_{0}\right)$ or if $s=s_{0}$, the following representations of $f_{k}(s)$ are valid:

$$
\begin{aligned}
f_{k}(s)= & \int_{0}^{\infty} \frac{\left(s-s_{0}\right)^{k+2}}{k !} e^{-\left(s-s_{0}\right) t}\left\{\left[\int_{0}^{t} e^{-s_{0} u} d F(u)-f_{k}\left(s_{0}\right)\right] * t^{k}\right\} d t \\
& +f_{k}\left(s_{0}\right),
\end{aligned}
$$




$$
\begin{aligned}
f_{k}(s)= & \int_{0}^{\infty} \frac{\left(s-s_{0}\right)^{k+1}}{k !} e^{-\left(s-s_{0}\right) t} d\left\{\left[\int_{0}^{t} e^{-s_{0} u} d F(u)-f_{k}\left(s_{0}\right)\right] * t^{k}\right\} \\
& +f_{k}\left(s_{0}\right) .
\end{aligned}
$$

The integrals in (5) and (6) are uniformly convergent in every region $Q_{w}\left(s_{0}\right)$. The integral in (5) is absolutely convergent for $R(s)>R\left(s_{0}\right)$.

Proof. Assertions of the theorem for the case $s=s_{0}$ can be easily verified. We therefore assume $s \neq s_{0}$. Reflection now reveals that it is immaterial whether we consider $s$ to be such that $R(s)>R\left(s_{0}\right)$ or whether we consider it to be such that it lies in some region $Q_{w}\left(s_{0}\right)$ $-\left\{s_{0}\right\}$. We therefore further assume that $s$ is in some region $Q_{w}\left(s_{0}\right)$ $-\left\{s_{0}\right\}$.

By Theorem $6 \mathrm{~b}$ of Widder ${ }^{2}$ and (3) we may write, since $R(s)>R\left(s_{0}\right)$,

$$
\int_{0}^{t} e^{-s u} d F(u)=\int_{0}^{t} e^{-\left(s-s_{0}\right) u} d G(u)
$$

From this it follows that

$$
M_{k}(s, t)=\frac{k}{t^{k}} \int_{0}^{t}\left\{\int_{0}^{u} e^{-\left(s-s_{0}\right) v} d G(v)\right\}(t-u)^{k-1} d u,
$$

or, since the Stieltjes integral with respect to a constant is 0 ,

$$
M_{k}(s, t)=\frac{k}{t^{k}} \int_{0}^{t}\left\{\int_{0}^{u} e^{-\left(s-s_{0}\right) v} d\left[G(v)-f_{k}\left(s_{0}\right)\right]\right\}(t-u)^{k-1} d u .
$$

After integrating by parts, using a method of Doetsch's ${ }^{3}$ and referring to (4) we get

$$
\begin{aligned}
M_{k}(s, t)= & \frac{e^{-\left(s-s_{0}\right) t}}{t^{k}} H_{1}(t)+\frac{\left(s-s_{0}\right) e^{-\left(s-s_{0}\right) t}}{k t^{k}} H_{2}(t) \\
& +\frac{1}{t^{k}} \int_{0}^{t} I_{1}(u, t) e^{-\left(s-s_{0}\right) u} H_{1}(u) d u \\
& +\frac{1}{k t^{k}} \int_{0}^{t} I_{2}(u, t) e^{-\left(s-s_{0}\right) u} H_{2}(u) d u \\
& +\frac{1}{k(k !)} \int_{0}^{t}\left(s-s_{0}\right)^{k+2} e^{-\left(\bullet-s_{0}\right) u} H_{2}(u) d u+f_{k}\left(s_{0}\right)
\end{aligned}
$$

where

2 David Vernon Widder, The Laplace transform, Princeton, Princeton University Press, 1946, p. 12.

${ }^{3}$ Doetsch, op. cit., pp. 316-317. 


$$
\begin{aligned}
I_{i}(u, t) & =\sum_{h=1}^{k-1+i}\left(\begin{array}{c}
k-1+i \\
h
\end{array}\right) \frac{\left(s-s_{0}\right)^{h-1+i}}{(h-1) !} \\
& \cdot \sum_{a+b=h-1, a \neq k}\left(\begin{array}{c}
h-1 \\
a
\end{array}\right) t^{a}(-u)^{b},
\end{aligned} \quad i=1,2 .
$$

By Theorem 2 the first two terms of (7) tend to 0 uniformly in $Q_{w}\left(s_{0}\right)$ as $t \rightarrow+\infty$. Using (8), property (2), Theorem 2, and a method of Doetsch's ${ }^{4}$ it can be shown that the third and fourth terms of (7) tend uniformly to 0 in $Q_{w}\left(s_{0}\right)$ as $t \rightarrow+\infty$. By Satz 4 of Doetsch Dend $^{5}$ and (2) the fifth term of (7) tends, as $t \rightarrow+\infty$, to the existing integral

$$
\frac{1}{k(k !)} \int_{0}^{\infty}\left(s-s_{0}\right)^{k+2} e^{-\left(s-s_{0}\right) t} H_{2}(t) d t,
$$

which converges uniformly in $Q_{w}\left(s_{0}\right)$. Substituting from (1) into (9) we get (5). That the integral in (5) converges absolutely for $R(s)$ $>R\left(s_{0}\right)$ follows from (1) and the order relation (2). Assertions about (6) follow from preceding results, (2), and Theorem 2.

CoROLlary 3.1. If $f_{k}$ exists, then the domain of $f_{k}$ is a half-plane or the whole plane.

Corollary 3.2. Let $f_{k}\left(s_{0}\right)$ exist and

$$
R(s)>R\left(s_{0}\right) \text {. }
$$

Then $f_{k}(s)$ exists and the following representations of $f_{k}(s)$ are valid:

$$
\begin{aligned}
& f_{k}(s)=\frac{\left(s-s_{0}\right)^{k+2}}{k !} \int_{0}^{\infty} e^{-\left(s_{-} s_{0}\right) t}\left\{\left[\int_{0}^{t} e^{-s_{0} u} d F(u)\right] * t^{k}\right\} d t, \\
& f_{k}(s)=\frac{\left(s-s_{0}\right)^{k+1}}{k !} \int_{0}^{\infty} e^{-\left(s-s_{0}\right) t} d\left\{\left[\int_{0}^{t} e^{-s_{0} u} d F(u)\right] * t^{k}\right\},
\end{aligned}
$$

where the integral in (10) converges absolutely for $R(s)>R\left(s_{0}\right)$.

THEOREM 4. If $f_{k}\left(s_{0}\right)$ exists and if $R(s)>R\left(s_{0}\right)$ or if $s=s_{0}$, then $f_{k}(s)$ exists and the following representations of $f_{k}(s)$ are valid:

$$
\begin{aligned}
f_{k}(s)= & \int_{0}^{\infty} \frac{\left(s-s_{0}\right)^{k+1}}{(k-1) !} e^{-\left(s_{-}-s_{0}\right) t} \\
& \cdot\left\{\left[\int_{0}^{t} e^{-s_{0} u} d F(u)-f_{k}\left(s_{0}\right)\right] * t^{k-1}\right\} d t+f_{k}\left(s_{0}\right),
\end{aligned}
$$

4 Ibid., p. 319.

Ibid., p. 34. 


$$
\begin{aligned}
f_{k}(s)= & \int_{0}^{\infty} \frac{\left(s-s_{0}\right)^{k}}{(k-1) !} e^{-\left(\bullet-s_{0}\right) t} \\
& \cdot d\left\{\left[\int_{0}^{t} e^{-s_{0} u} d F(u)-f_{k}\left(s_{0}\right)\right] * t^{k-1}\right\}+f_{k}\left(s_{0}\right),
\end{aligned}
$$

the integrals in (12) and (13) being uniformly convergent in every region $Q_{w}\left(s_{0}\right)$. The integral in (12) is absolutely convergent for $R(s)>R\left(s_{0}\right)$.

Proof. By (5) of Theorem 3 and the notation introduced in (1) we may write for $s$ in any region $Q_{w}\left(s_{0}\right)$, or for any $s$ such that $R(s)$ $>R\left(s_{0}\right)$ or $s=s_{0}$,

$$
f_{k}(s)=\int_{0}^{\infty} \frac{\left(s-s_{0}\right)^{k+2}}{k !} e^{-\left(s-s_{0}\right) t}\left\{\frac{H_{2}(t)}{k}\right\} d t+f_{k}\left(s_{0}\right) .
$$

But since, by (1) and (2),

$$
\begin{aligned}
\frac{H_{2}(t)}{k} & =k\left\{\left[\int_{0}^{t} e^{-s_{0} u} d F(u)-f_{k}\left(s_{0}\right)\right] * t^{k-1}\right\} * 1 \\
& =\int_{0}^{t} H_{1}(u) d u=o\left(t^{k+1}\right)
\end{aligned}
$$

as $t \rightarrow+\infty$, we can apply Satz 2 of Doetsch, ${ }^{6}$ Satz 4 of Doetsch, ${ }^{7}(1)$, and (2) to obtain (12) from (14), where the integral in (12) converges uniformly in $Q_{w}\left(s_{0}\right)$. The absolute convergence of the integral in (12) for $R(s)>R\left(s_{0}\right)$ follows from a consideration of (2). The uniform convergence in $Q_{w}\left(s_{0}\right)$ of the integral in (13) follows as in the proof of Theorem 3. The theorem is thus proved.

Corollary 4.1. Let $f_{k}\left(s_{0}\right)$ exist and $R(s)>R\left(s_{0}\right)$. Then $f_{k}(s)$ exists and the following representations of $f_{k}(s)$ are valid:

$$
\begin{aligned}
& f_{k}(s)=\frac{\left(s-s_{0}\right)^{k+1}}{(k-1) !} \int_{0}^{\infty} e^{-\left(s-s_{0}\right) t}\left\{\left[\int_{0}^{t} e^{-s_{0} u} d F(u)\right] * t^{k-1}\right\} d t \\
& \left.f_{k}(s)=\frac{\left(s-s_{0}\right)^{k}}{(k-1) !} \int_{0}^{\infty} e^{-\left(s-s_{0}\right) t} d\left[\int_{0}^{t} e^{-s_{0} u} d F(u)\right] * t^{k-1}\right\},
\end{aligned}
$$

where the integral in (15) converges absolutely for $R(s)>R\left(s_{0}\right)$.

The proof of the next theorem follows by writing, for $k \geqq 2$,

6 Ibid., p. 90.

7 Ibid., p. 34. 


$$
\begin{aligned}
& {\left[\int_{0}^{t} e^{-s_{0} u} d F(u)-f_{k}\left(s_{0}\right)\right] * t^{k-1}} \\
& \quad=(k-1) \int_{0}^{t}\left\{\left[\int_{0}^{u} e^{-s 0 v} d F(v)-f_{k}\left(s_{0}\right)\right] * u^{k-2}\right\} d u,
\end{aligned}
$$

and then applying (12) of Theorem 4, (2), and Satz 2 of Doetsch:8

THEOREM 5. Let $f_{k}\left(s_{0}\right)$ exist and $k \geqq 2$. Then $f_{k}(s)$ exists for $R(s)$ $>R\left(s_{0}\right)$. If $R(s)>R\left(s_{0}\right)$ or if $s=s_{0}$, the following representation of $f_{k}(s)$ is valid:

$$
\begin{aligned}
f_{k}(s)= & \int_{0}^{\infty} \frac{\left(s-s_{0}\right)^{k}}{(k-2) !} e^{-\left(s-s_{0}\right) t}\left\{\left[\int_{0}^{t} e^{-s_{0} u} d F(u)-f_{k}\left(s_{0}\right)\right] * t^{k-2}\right\} d t \\
& +f_{k}\left(s_{0}\right) .
\end{aligned}
$$

If $R(s)>R\left(s_{0}\right)$, then also

$$
f_{k}(s)=\frac{\left(s-s_{0}\right)^{k}}{(k-2) !} \int_{0}^{\infty} e^{-\left(s-s_{0}\right) t}\left\{\left[\int_{0}^{t} e^{-s_{0} u} d F(u)\right] * t^{k-2}\right\} d t .
$$

4. Function theoretic properties of the transform. In this section we use the notation $L^{(k)}(F)=f_{k}$.

THEOREM 6. Let $F_{1}$ and $F_{2}$ be two functions from the class $\{F\}$ such that $F_{1}(0)=F_{2}(0)$ and such that

$$
L^{(k)}\left(F_{1}\right)=L^{(k)}\left(F_{2}\right)=f_{k} .
$$

Then $F_{1}(t)=F_{2}(t)$ almost everywhere for $t \geqq 0$.

Proof. Since $f_{k}$ exists, there is a real $s_{0}>0$ such that for all $s$ where $R(s)>s_{0} f_{k}(s)$ is given by (15). Defining

$$
G_{i}(t)=\int_{0}^{t} e^{-s 0 u} d F_{i}(u), \quad i=1,2,
$$

we easily get from (15), for all $s$ where $R(s)>s_{0}$,

$$
\int_{0}^{\infty} e^{-\left(s-s_{0}\right) t}\left\{\left[G_{1}(t)-G_{2}(t)\right] * t^{k-1}\right\} d t=0 .
$$

By Theorem $5 \mathrm{c}$ of Widder ${ }^{9} G_{1}(t)-G_{2}(t)$ is of bounded variation on every closed interval $[0, T], T \geqq 0$ and by Satz 4 of Doetsch ${ }^{10}$

${ }^{8}$ Ibid., p. 90.

Op. cit., p. 9.

10 Op. cit., p. 113. 
$\left[G_{1}(t)-G_{2}(t)\right]^{*} t^{k-1}$ is a $J$-Function "in the sense of Doetsch." ${ }^{11} \mathrm{By}$ Satz 4, of Doetsch ${ }^{12}$ Satz 12 of Doetsch, ${ }^{13}$ and Theorem 11.52 of Titchmarsh ${ }^{14}$ we get that $G_{1}(t)=G_{2}(t)$ almost everywhere for $t \geqq 0$. Referring to (19) and (20) we may now write

$$
\int_{0}^{t} e^{-s 0 v} d\left[F_{1}(v)-F_{2}(v)\right]=0
$$

almost everywhere for $t \geqq 0$. Integrating (21) by parts and using the hypothesis $F_{1}(0)=F_{2}(0)$ we get

$$
e^{-s_{0} t}\left[F_{1}(t)-F_{2}(t)\right]+s_{0} \int_{0}^{t} e^{-s_{0} v}\left[F_{1}(v)-F_{2}(v)\right] d v=0
$$

almost everywhere for $t \geqq 0$. Theorem 6 now follows easily from (22).

Theorem 6 now yields the following theorem:

TheOREM 7. Let $F_{1}$ and $F_{2}$ be two functions from the class $\{F\}$ such that

$$
L^{(k)}\left(F_{1}\right)=L^{(k)}\left(F_{2}\right)=f_{k} .
$$

Then $F_{1}(t)=F_{2}(t)+\left[F_{1}(0)-F_{2}(0)\right]$ almost everywhere for $t \geqq 0$.

THEOREM 8. Let $f_{k}\left(s_{0}\right)$ exist. Then $f_{k}$ is analytic in the region defined by $R(s)>R\left(s_{0}\right)$.

Proof. The theorem follows from the fact that $f_{k}(s)$ is representable by a Laplace or Laplace-Stieltjes integral if $R(s)>R\left(s_{0}\right)$.

THEOREM 9. $f_{k}$ is analytic in the half-plane, or the whole plane, of its existence.

Proof. The theorem follows from Corollary 3.1 and Theorem 8 .

The following theorem is easily proved:

THEOREM 10. $M_{k}(s, t)$ is an integral function of $s$.

Theorem 11. Let $f_{k}\left(s_{0}\right)$ exist. Then

$$
f_{k}\left(s_{\alpha}\right) \rightarrow f_{k}\left(s_{0}\right)
$$

if $\left\{s_{\alpha}\right\}$ is any sequence in any region $Q_{w}\left(s_{0}\right)$ such that $\left\{s_{\alpha}\right\} \rightarrow s_{0}$.

Proof. The theorem follows from Theorem 3 and Theorem 10.

11 Ibid., p. 29.

12 Ibid., p. 74.

${ }^{13}$ Ibid., p. 131.

${ }^{14} \mathrm{E}$. C. Titchmarsh, The theory of functions, 2d ed. London, Oxford University Press, 1939, p. 360. 
TheOREM 12. If $f_{k}\left(s_{0}\right)$ exists, then for any $c>R\left(s_{0}\right)$ and $t \geqq 0$

$$
\left[\int_{0}^{t} e^{-s_{0} u} d F(u)\right] * t^{k-1}=\operatorname{Lim}_{T \rightarrow+\infty} \frac{(k-1) !}{2 \pi i} \int_{c-i T}^{c+i T} \frac{f_{k}(s)}{\left(s-s_{0}\right)^{k+1}} e^{s t} d s .
$$

Proof. We assume $f_{k}\left(s_{0}\right)$ exists, $c>R\left(s_{0}\right)$, and $t \geqq 0$. Let

$$
H(t)=\left[\int_{0}^{t} e^{-s 0 u} d F(u)\right] * t^{k-1} .
$$

By (15) of Corollary 4.1 and (23) we have

$$
f_{k}(s)=\frac{\left(s-s_{0}\right)^{k+1}}{(k-1) !} \int_{0}^{\infty} e^{-\left(s-s_{0}\right) t} H(t) d t,
$$

where the integral converges absolutely for $R(s)>R\left(s_{0}\right)$. By reasoning similar to that in the proof of Theorem 6 we can assert that $H$ is a $J$-Function "in the sense of Doetsch," 15 and that $H$ is continuous and of bounded variation on every finite, closed interval $[0, T], T \geqq 0$. Hence we can apply Satz 3 of Doetsch ${ }^{16}$ to

$$
L(H)=\frac{(k-1) ! f_{k}(s)}{\left(s-s_{0}\right)^{k+1}}
$$

to obtain

$$
\left[\int_{0}^{t} e^{-s 0 u} d F(u)\right] * t^{k-1}=\operatorname{Lim}_{T \rightarrow+\infty} \frac{(k-1) !}{2 \pi i} \int_{c-i T}^{c+i T} \frac{f_{k}(s)}{\left(s-s_{0}\right)^{k+1}} e^{s t} d s .
$$

UNIVERSITY OF COLORADO

15 Op. cit., p. 29.

16 Ibid., p. 212. 